\title{
Safety and Efficacy of Collagen-Based Biological Dressings in the Management of Chronic Superficial Skin Wounds in Non-Complex Trauma: A Post-Marketing Surveillance Study
}

\author{
Francesco De Francesco ${ }^{1, *} \mathbb{0}$, Marialuisa De Francesco ${ }^{2}$ and Michele Riccio ${ }^{1}$ \\ 1 Department of Plastic Reconstructive Surgery and Hand Surgery, Azienda Ospedaliero Universitaria \\ Ospedali Riuniti, 60126 Ancona, Italy; michele.riccio@ospedaliriuniti.marche.it \\ 2 Data Analysis Office, University of Milan, 20122 Milan, Italy; Marialuisa.DeFrancesco@unimi.it \\ * Correspondence: francesco.defrancesco@ospedaliriuniti.marche.it; Tel.: +39-071-596-3945
}

Citation: De Francesco, F.; De

Francesco, M.; Riccio, M. Safety and Efficacy of Collagen-Based Biological Dressings in the Management of Chronic Superficial Skin Wounds in Non-Complex Trauma: A Post-

Marketing Surveillance Study. Trauma Care 2021, 1, 195-205. https:// doi.org/10.3390/traumacare1030017

Received: 29 June 2021

Accepted: 27 November 2021

Published: 29 November 2021

Publisher's Note: MDPI stays neutral with regard to jurisdictional claims in published maps and institutional affiliations.

Copyright: (c) 2021 by the authors. Licensee MDPI, Basel, Switzerland. This article is an open access article distributed under the terms and conditions of the Creative Commons Attribution (CC BY) license (https:/ / creativecommons.org/licenses/by/ $4.0 /)$.

\begin{abstract}
Biological dressings such as collagen and hyaluronic acid represent the main advanced tools that plastic surgeons, dermatologists and vulnologists use today to treat chronic wounds or ulcers that do not tend to heal. These types of dressings are important because they create a moist and physiological interface at the wound level, are of natural origin, easy to use, hypo-allergenic, economical and do not create discomfort for the patient during dressing changes. We treated 128 patients (divided into four groups based on type of dressing) with non-complex superficial chronic wounds in comparison with a traditional dressing (fitostimoline gauze or polyurethane foam). We analyzed wound characteristics, healing time, and operator and patient satisfaction. A significantly higher recovery rate was observed in the "Collagen-coated plates" treatment group compared to Standard Treatment. Additionally, a significantly higher probability of recovery was observed compared to the alternative two experimental devices (Collagen-coated plates + HA and Collagen-based spray). However, the main limitation of the randomization of this study is the presence in the "Collagen-based spray" group of localized wounds, mainly in the fingers and hand. No side effects were reported in relation to the procedures or the experimental products. Collagen may be considered as a valuable therapeutic tool in non-complex chronic wounds by virtue of its low immunogenicity, flexibility and applicability in biocompatible scaffolds, and represents driving force toward enhanced wound care.
\end{abstract}

Keywords: advanced dressings; biological dressings; collagen sponge; hyaluronic acid sponge; superficial skin wound

\section{Introduction}

Chronic wounds commonly involve delayed healing processes, and the incidence rate of challenging recoveries is on the rise due to the ageing population and an increase in risk factors such as diabetes, obesity, and hypertension. Chronic wounds represent a significant clinical and economic burden, with an annual treatment cost of over USD 20 billion; some two million people suffer from this type of wound in the United States $[1,2]$. In Italy, approximately two million people are affected by complex wounds, costing the National Health System EUR 1.5 billion yearly. The incidence rate is expected to increase by $8 \%$ within the next few years on account of the aforementioned risk factors of diabetes, obesity and vascular disorders. A wound is defined as a discontinuity of one or more bodily tissues, which may differ based on the mechanism of injury and healing time. Moreover, wounds may be classified as acute lesions, which heal within 6 to 8 weeks, or as chronic, characterized by the failure of normal phases of wound healing in an orderly and timely manner, with a recovery time that usually exceeds 8 weeks.

The mechanism of injury will determine a shallow wound with dermis remaining intact or a deep wound involving the dermis $[3,4]$. 
Standard treatment (ST) includes wound cleansing or debridement, antiseptic treatment, and management of infection. Cleansing or debridement of wounds and removal of debris and contaminants are fundamental interventions which may be performed on an outpatient basis in cases of superficial lesions or as an inpatient requiring surgical treatment in cases of deep and particularly contaminated wounds. The type of medication will vary according to the recommended treatments currently available and the wound type. Treatments are generally classified as conventional and advanced microbial-based or non-microbial therapies. Additionally, conventional medication may be defined as primary or acting directly on the wounded area, playing a protective role as well as a haemostatic function, supplying coverage and absorption (Fitostimoline gauze or polyurethane foam), while advanced therapy involves biocompatible dressing coverage providing a moist environment between the wound and medication. According to a meta-analysis conducted on ten control groups in a randomized-controlled study (RCTs), $<30 \%$ of the patients affected by a complex wound will recover within twenty weeks of standard treatment (ST) initiation. Furthermore, a more recent randomized-controlled study reports an average healing time of 57.4 days in wounds treated with ST alone, compared to 23.5 days in wounds treated with EpiFix (MiMedx, Marietta, GA, USA) [5] and 47.9 days for wounds treated with a double-layered bioengineered cutaneous substitute (Apligraf; Canton, MA, USA) [6]. These findings suggest that standard treatment alone is unable to ensure rapid healing and will entail longer hospital stays and potential contamination exposure in patients with unresolved wounds.

The extracellular matrix (ECM) is destroyed and unable to sustain healing in superficial and deep wounds, hence the need to resort to pre-planned treatment strategies to substitute the lack or restore the functions of ECM [7]. A moist healing environment in the wound area is fundamental to endorse regeneration of damaged tissues and to prevent the formation of eschar, and will stimulate growth factors, fibroblasts, keratinocytes as well as the inflammatory response and phagocyte activation [8,9]. Numerous investigations confirm that collagen-based products generate a steady and moist healing environment and promote the recovery process in chronic or acute cutaneous wounds of different etiologies. The repairing process of the wound is rapid, and the number of applications is reduced, which consequently diminishes the incidence of wound dehiscence and local signs of inflammation $[2,10]$.

Administration of safe and reliable medication is of utmost importance to stimulate the recovery process as well as supplying an efficient protective barrier to preclude microbial cross-contamination. Numerous clinical trials assessing the efficacy of collagen-based products confirm their ability to maintain the wound environment free from external microbial contamination, thus regulating the bacterial status of chronic or acute wounds of different aetiologies [11] and ensuring a moist wound environment that favours rapid re-epithelialisation. Healing time is significantly reduced in collagen-based medications compared to standard treatments, diminishing the risk of wound maceration. Some dressings containing heterologous equine collagen Type I may promote recovery in the granulation stage of acute wound healing [12].

The aim of this study is to assess the efficacy of three "advanced" collagen-based medications (Euroresearch, Milan, Italy) compared to ST in treating chronic superficial cutaneous wounds. These medications contain $90 \%$ of native collagen and provide a three-dimensional (3D) native structure which may support important cell proliferation for tissue repair. In addition, the flexible properties of the product and the three-dimensional open-pore materials should permit the collagen to act as a scaffold for the various types of cells involved in wound healing granulation and subsequent tissue growth.

We also evaluate collagen-based devices and their potential to accelerate the recovery process in superficial skin wounds by calculating the rate of re-epithelialisation and the average percentage reduction of the wound area. 


\section{Materials and Methods}

A single-centre randomized prospective study was performed in order to assess the safety and efficacy of three medical device-based Equine collagens: Group A, "Sterile Collagen-coated plates + HA"; Group B, "Sterile Collagen-based spray $75 \mathrm{~mL}$ bottles"; and Group C, "Sterile collagen-based plates", in relation to the recovery rate of superficial skin wounds. Every group was compared to the standard treatment, Group D, "Fitostimoline gauze or polyurethane foam".

The study was performed in compliance with the Declaration of Helsinki and the Guidelines for Good Clinical Practice. The patients provided written informed consent for both treatment and follow-up, then underwent screening to determine eligibility for the study according to the inclusion and exclusion criteria set in Table 1.

Table 1. Inclusion and exclusion criteria.

\begin{tabular}{|c|c|}
\hline Inclusion Criteria & Exclusion Criteria \\
\hline Non-complex chronic wound ( $>8$ weeks) & Pregnancy and/or Breast-feeding \\
\hline Age $\geq 18$ and $\leq 85$ years & Non-superficial wound with exposed bone, tendon or muscle fascia \\
\hline Superficial wound of different aetiologies & $\begin{array}{l}\text { WBS score less than or equal to } 14 \text { (abundant exudation, perilesional } \\
\text { dermatitis) }\end{array}$ \\
\hline $\begin{array}{l}\text { Wound bed score }>15 \text { (WBS) (Falanga, 2016) } \\
\text { Non-infected wound according to CDC/NHSN }\end{array}$ & Signs of infection according to CDC/NHSN \\
\hline $\begin{array}{l}\text { Surveillance definitions } \\
\text { Wound dimension range }\left(5-80 \mathrm{~cm}^{2}\right)\end{array}$ & Presence of fistula \\
\hline Written consent & $\begin{array}{l}\text { Connective tissue disorders i.e., Systemic Lupus, Systemic sclerosis, } \\
\text { Sjogren's syndrome } \\
\text { Active sickle-cell anemia } \\
\text { Corticosteroid-induced, chemotherapy-induced immunosuppression, } \\
\text { use of antiangiogenic agents or immunosuppressants, } \\
\text { immune-compromised status related to immunodeficiency disorders } \\
\text { Hypersensitivity to active substances or excipients of medications }\end{array}$ \\
\hline
\end{tabular}

The follow-up study protocol was approved by the local Ethics Committee (2020/56 on 7 May 2020). Demographic data, medical history and wound measurements were collected (Table 2).

Table 2. Demographic data of patients.

\begin{tabular}{ccccc}
\hline & Group A & Group B & Group C & Group D \\
\hline Gender & & & & \\
Male & $12(80 \%)$ & $11(74 \%)$ & $10(71 \%)$ & $12(79 \%)$ \\
Female & $3(20 \%)$ & $4(26 \%)$ & $5(29 \%)$ & $3(21 \%)$ \\
Age & & & & \\
Average & 59.1 & 56.9 & 58.5 & 62.3 \\
Median & 58 & 56 & 60.5 & 60 \\
SD & 12.76 & 10.86 & 9.83 & 37.43 \\
Range & $24-85$ & $33-85$ & $34-80$ & \\
Smokers & & & & $12(80 \%)$ \\
Yes & $10(65 \%)$ & $8(51 \%)$ & $6(40 \%)$ & $3(20 \%)$ \\
No & $5(35 \%)$ & $7(49 \%)$ & $9(60 \%)$ & 33.6 \\
BMI & & & & 34 \\
Average & 32.56 & 32.82 & 31.41 & 7.773 \\
Median & 31.7 & 31.5 & 32.1 & $21-45$ \\
SD & 8.276 & 6.929 & 5.082 & $9(60 \%)$ \\
Range & $19.9-81.6$ & $19.5-50.2$ & $23.4-44.2$ & $11(70 \%)$ \\
Comorbidity & & & & $6(40 \%)$ \\
Diabetes & $10(65 \%)$ & $9(60 \%)$ & $10(66 \%)$ & $11(68 \%)$ \\
Hypertension & $10(65 \%)$ & $11(69 \%)$ & $5(36 \%)$ & \\
Dyslipidemia & $5(35 \%)$ & $4(31 \%)$ &
\end{tabular}




\subsection{Study Design}

The patients who met the inclusion criteria were randomly assigned to one of the following four treatment arms: Sterile collagen-coated plates + HA (Group A); Sterile Collagen-based spray in $75 \mathrm{~mL}$ bottles (Group B); Sterile Collagen-coated plates (Group C); standard treatment alone, Fitostimoline gauze (Group D) with a 1:1:1:1 ratio. Primary and secondary endpoints were detected at V2: 7 days; V3: 14 days; V4:21 days; V5: 28 days and compared to basal examination (V1: 0 day).

Eligible patients were assigned to the first random number according to the medical device available in the centre. None of the patients withdrew consent following randomization and treatment initiation. Two randomized patients were excluded from the study following complications related to Sterile, in Group A. The primary endpoint was acceleration of the recovery process in superficial skin wounds compared to standard treatment alone, considering the re-epithelialisation rate (average percentage decrease of wound area in relation to basal area) following 14 days of treatment.

Secondary endpoints included rate of re-epithelialisation (average percentage decrease of wound area in relation to basal area) at different time intervals; complete wound recovery and re-epithelialisation percentage at all time intervals; protection efficacy in bacterial infections (uninfected wounds); patient satisfaction with wound appearance; global clinical assessment of wound; and the safety and tolerability of treatments. Complete wound closure was defined as re-epithelialisation of $100 \%$ without medication (confirmed by two consecutive examinations two weeks apart) in accordance with regulations set by the Agency for Healthcare Research and Quality (AHRQ, Rockville, MA, USA) (2011). Safety measurements were retained as necessary following the increased frequency of serious adverse events (SAEs).

\subsection{Standard Treatment}

Patients were prepared and wounds were primarily covered with a tulle gras dressing, ensuring painless removal. The tulle gras medication is not absorbent, necessitating secondary medication to absorb blood and exudates from the wound. A sterile gauze was thus placed onto the tulle Gras medication. The primary and secondary medications were applied to cover the injured area generously to avoid peri-wound maceration. At weekly intervals (V2-V5), patient assessment was conducted in the enrolment centres containing records of wound size and signs of infection. Investigators conducted wound medication and dressing change on examination.

\subsection{Experimental Treatment}

Patients randomly assigned to Groups A, B and C were required to apply Equine Collagen as per medical prescription on the wound after wound bed preparation At weekly intervals (V2-V5), patient assessment was conducted in the enrolment centres containing records of wound size and signs of infection. Investigators conducted wound medication and dressing change on examination.

\subsection{Assessment Methods}

Patients were assessed weekly at V1-V5. The wound surface area was measured and recorded on each examination. Changes in wound size are difficult to measure accurately by length and width due to their irregular shape; hence, we measured the surface area in $\mathrm{cm}^{2}$ using squared and transparent paper on which we drew the wound area with indelible marker pens (Oxford Health NHS Foundation Trust guidelines, Oxford, UK). On wound closure, a second assessment was conducted after two weeks to confirm complete healing of the wound and to exclude relapses, according to AHRQ recommendations. Complete wound closure observed on second physical assessment was recorded as wound recovery on first observation. If wound closure had not occurred during final assessment, weekly monitoring was prescribed until confirmation of complete wound closure and recovery. In any such cases, the investigator addressed and evaluated the delay in wound 
healing (i.e., infection, inflammation, allergic reaction or non-compliance of the medical device). Assessments were conducted to acquire information on wound area, granulation/epithelialisation and safety on each physical examination. Global assessment of the patient (PTGA) was conducted oat V2, V3, V4, and V5, and the patient was required to express aesthetic satisfaction evaluation using a five-point Likert scale: "much worse than expected", "worse than expected", "as expected", "better than expected", "much better than expected". Global clinical assessment of the observer (COGA) was conducted at $\mathrm{V} 2, \mathrm{~V} 3, \mathrm{~V} 4$, and V5, and the investigator was required to evaluate wound status using a five-scale set: 1 = deterioration, $2=$ no change, $3=$ slight improvement, $4=$ moderate improvement, 5 = significant improvement. Adverse events (AE) were recorded from study initiation to study termination. All adverse events were reported as separate events including signs, symptoms, laboratory findings or disease, and were monitored until complete recovery or conclusive physical examination and assessment.

\subsection{Statistical Analysis}

Measurements were conducted in relation to the groups, and data were analysed with the ANOVA test. Statistically significant differences were calculated by Student's $t$-test. The threshold for statistical difference was based on the $p$-value, significant at $<0.05$. Statistical analysis software (Excel Xlstat add-on component) was used to conduct the two-tailed Student's $t$-test along with the Pearson Index and the Kolmogorov-Smirnov test.

\section{Results}

A total of 128 patients with superficial skin wounds were enrolled in the study. Patients were randomly assigned to four groups, as follows: 32 patients to the "Sterile, Collagen-coated plates + HA" treatment arm (Group A), 32 patients to the "Sterile, Collagen-based Spray $75 \mathrm{~mL}$ bottle" treatment arm (Group B), 32 patients to the "Sterile, Collagen-coated plate" treatment arm (Group C) and 32 to the "Standard treatment" arm (Group D). Table 3 shows the characteristics of wound location, the basal wound area at V1, and the wound duration.

Table 3. Characteristics of basal wound area.

\begin{tabular}{ccccc}
\hline & Group A & Group B & Group C & Group D \\
\hline Wound location & & & & \\
Finger/Hand & $7(46.6 \%)$ & $15(100 \%)$ & $7(46.6 \%)$ & $6(40 \%)$ \\
Foot & $6(40 \%)$ & $0(0 \%)$ & $5(33.3 \%)$ & $7(46.6 \%)$ \\
Upper or Inf. Limb & $1(6.6 \%)$ & $0(0 \%)$ & $2(13.3 \%)$ & $3(20 \%)$ \\
Other & $1(6.6 \%)$ & $0(0 \%)$ & $1(6.6 \%)$ & $0(0 \%)$ \\
Basal wound area & & & & \\
size (cm ${ }^{2}$ ) & & 5,4 & 50 & 52 \\
Average & 43 & 5 & 50 & 55 \\
Median & 45 & 0.5 & 21.29 & 18.87 \\
SD & 12.36 & $5-6$ & $20-80$ & $20-80$ \\
Range & $20-80$ & & & \\
Basal wound & & 5 & 6 & 7 \\
duration (weeks) & & 5 & 6 & 7.463 \\
Average & 6 & 1.4 & 1.463 & $5.0-9.0$ \\
Median & 6 & $3.0-7.0$ & $4.0-8.0$ & \\
SD & 1.463 & & &
\end{tabular}

The average age of participants in Group A was 59.1, in group B 56.9, in group C 58.5, and in the standard treatment group D, 62.3 (Table 2). On average, patients in the four groups were $76.2 \%$ male, with an average body mass index of 32.6 . The study population included patients presenting co-morbidities, which were duly recorded. The main comorbid conditions reported were dyslipidemia $(30.5 \%)$, diabetes $(62.75 \%)$ and hypertension $(68.5 \%)$. The proportion of patients receiving hypertension therapy was $54 \%$, with diabetes management in $48 \%$. The smoking history of the patients was recorded: $25 \%$ were current 
or past smokers while $58.7 \%$ were non-smokers. No statistically significant differences were found among the groups; however, the main limitation of the randomization of this study is the presence in group B of localized wounds, mainly in the fingers and hands.

Healing time was reported for all treatment groups at four weeks and at two weeks follow-up. A significantly higher wound recovery rate was observed in Group C compared to Group D at V2 (65.0\% vs. $41.1 \% ; 95 \%$ confidence interval $(\mathrm{CI})=1.1-3.5 ; p=0.0123)$, at V3 (82.5\% vs. $48.1 \%$; $95 \% \mathrm{CI}=1.4-4.1 ; p=0.0003)$, at $\mathrm{V} 4(89.7 \%$ vs. $66.2 \% ; 95 \% \mathrm{CI}=1.2-3.9$; $p=0.003)$ and at V5 (93.5\% vs. $71.2 \% ; 95 \% \mathrm{CI}=1.2-4.1 ; p=0.002)$. Additionally, a significantly higher probability of recovery was observed compared to Group A and B at V2 $(65.0 \%$ vs. $51.1 \%$ vs. $45 \%$; $p=0.003)$, V3 $(82.5 \%$ vs. $68.1 \%$ vs. $63.2 \% ; p=0.0006)$, V4 $(89.7 \%$ vs. $77.3 \%$ vs. $74.2 \% ; p=0.0145)$ and V5 $(93.5 \%$ vs. $81.2 \%$ vs. $79.6 \% ; p=0.023)$ (Figure 1).

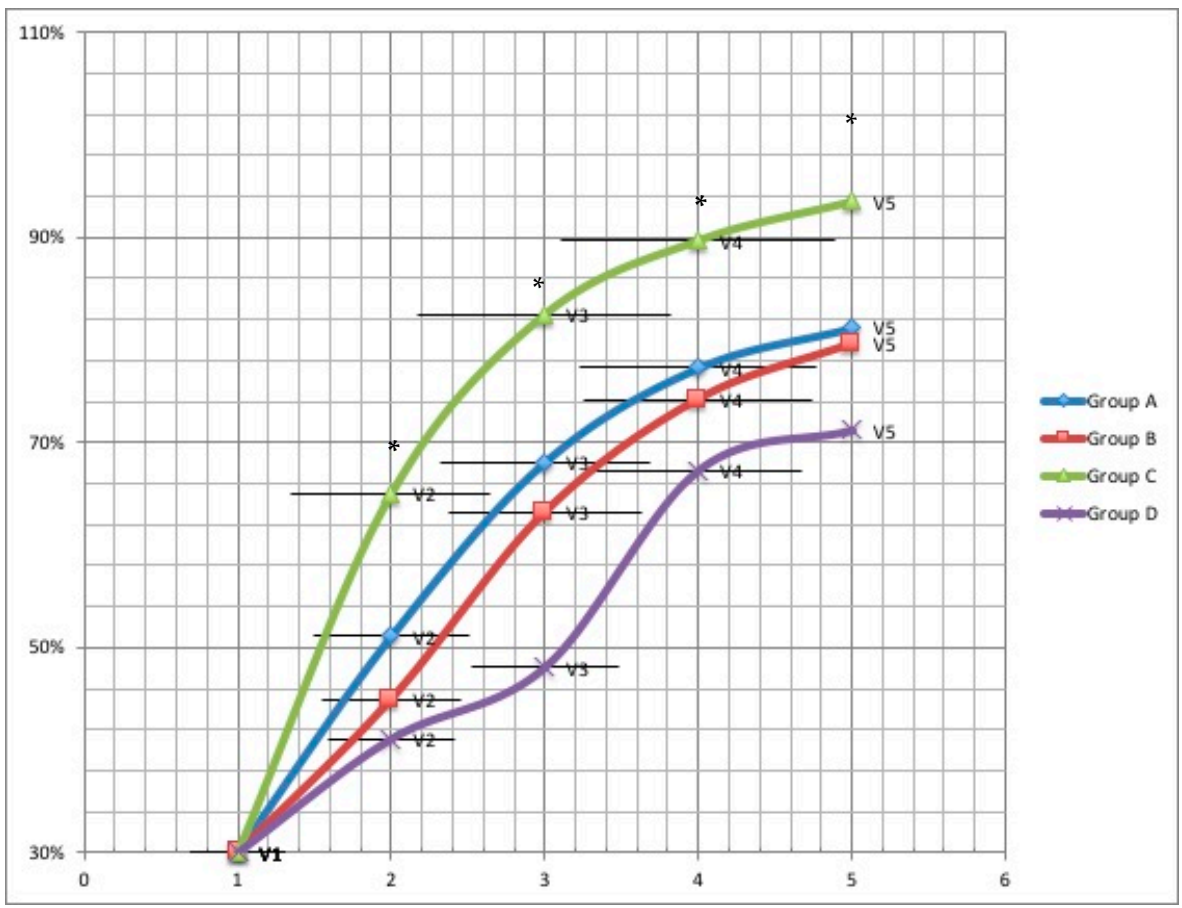

Figure 1. Wound healing rate over time. A significative difference was found between Group C compared to Group D in V2, V3, V5 $(p<0.01)$ *. Moreover, the wound healing rate was higher in group C compared to group A and B for all endpoints $(p<0.01)^{*}$.

In Group $C$, a significantly higher percentage of wound area reduction was observed compared to Group A, B, and D at V2, V3, V4 and V5. Sustained healing was observed in Group A, B, and C at a significantly higher rate than in the standard treatment (Group D) two weeks following termination $(100 \%$ vs. $86.7 \% ; p=0.0435)$.

Further investigations were conducted as secondary objectives, specifically patient satisfaction regarding wound outcomes (PTGA) and clinician's global assessment of the wound (COGA). The average global scores of PTGA on study termination were as follows: 4 for the Group A, 4 for Group B, 5 for Group C and 4 for Group D (Figure 2). No significant differences were observed among the medical device treatment arms $(p>0.05)$. 


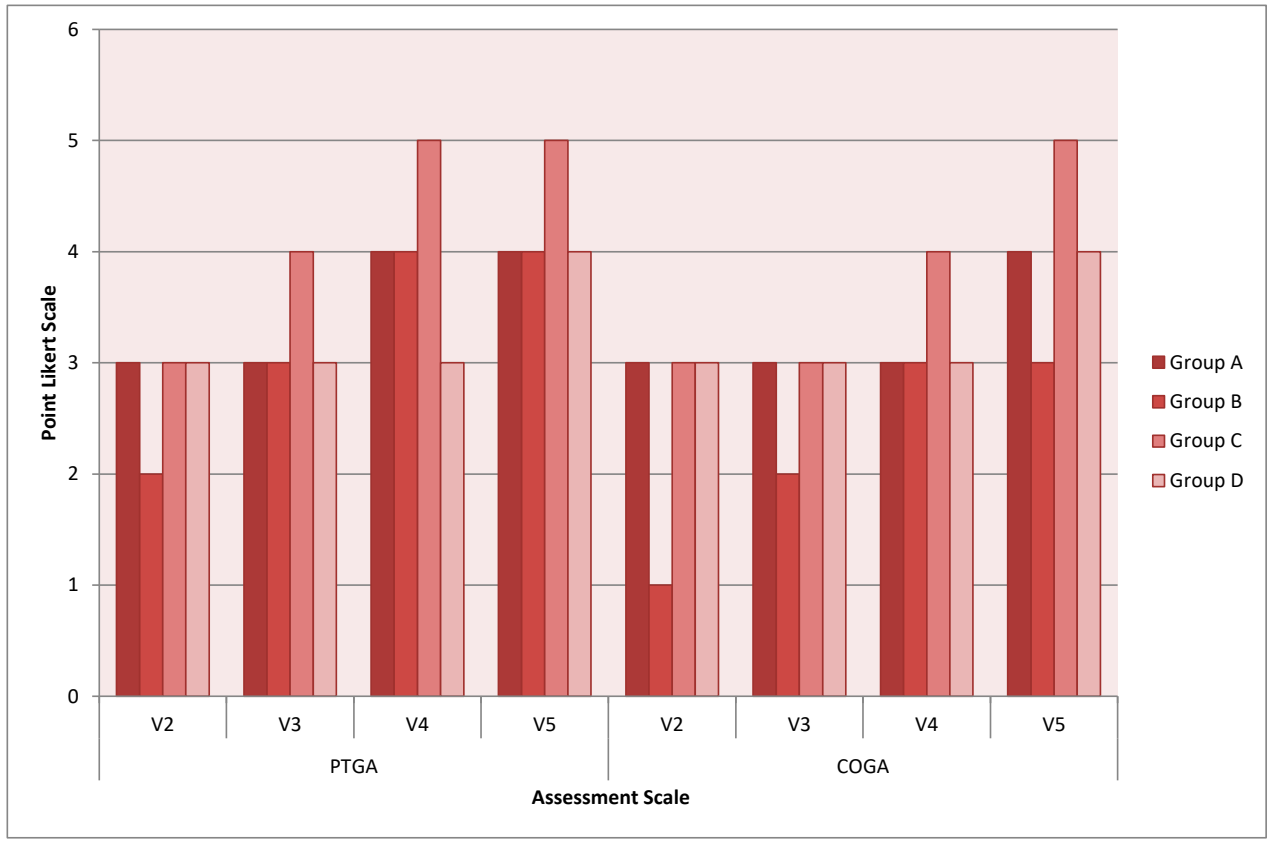

Figure 2. Average global assessment scale PTGA and COGA. The Likert 5-point scale did not show significative differences between any of the Groups at the different endpoints (V2-V5).

No AEs or SAEs were reported in relation to the procedures or to the experimental products. However, it is noteworthy that two participants were removed from the study due to complications associated with the medical device in Group A. The patients subsequently underwent advanced surgical intervention. Infections were also attentively monitored through microbiological analysis of cultures at V2, V3 and V4, with no suprainfection noted during the study course. Figures 3-6 show examples of clinical cases.

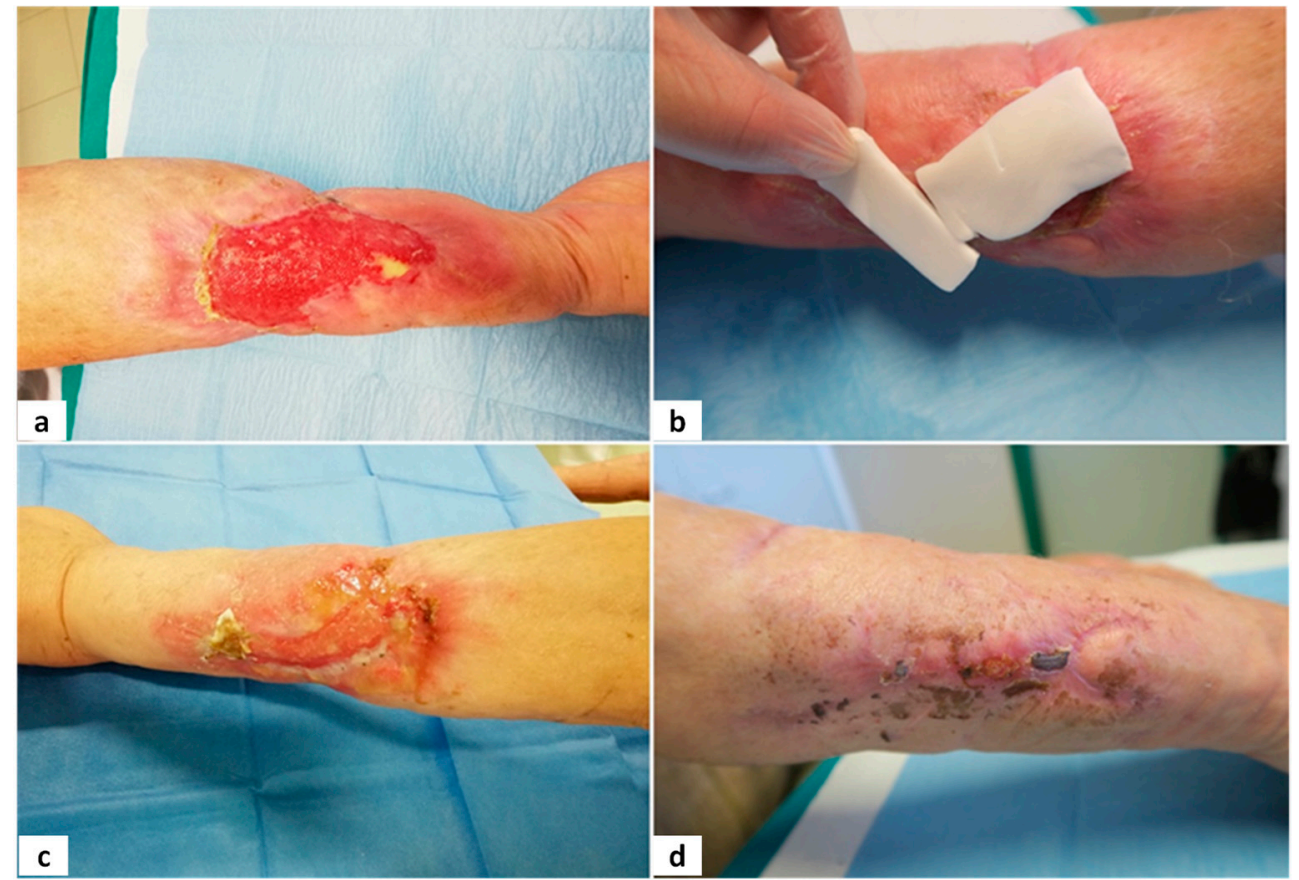

Figure 3. Case Group A. M.F, female 55 y/o affected by diabetes and hypertension reported a post-traumatic chronic wound in the dorsal part of the right arm close to the elbow (a). The aspect of the wound at V1 and the application of collagen plates enriched with hyaluronic acid (b). The wound aspect at V3 (c). The final aspect at V5 (d). 


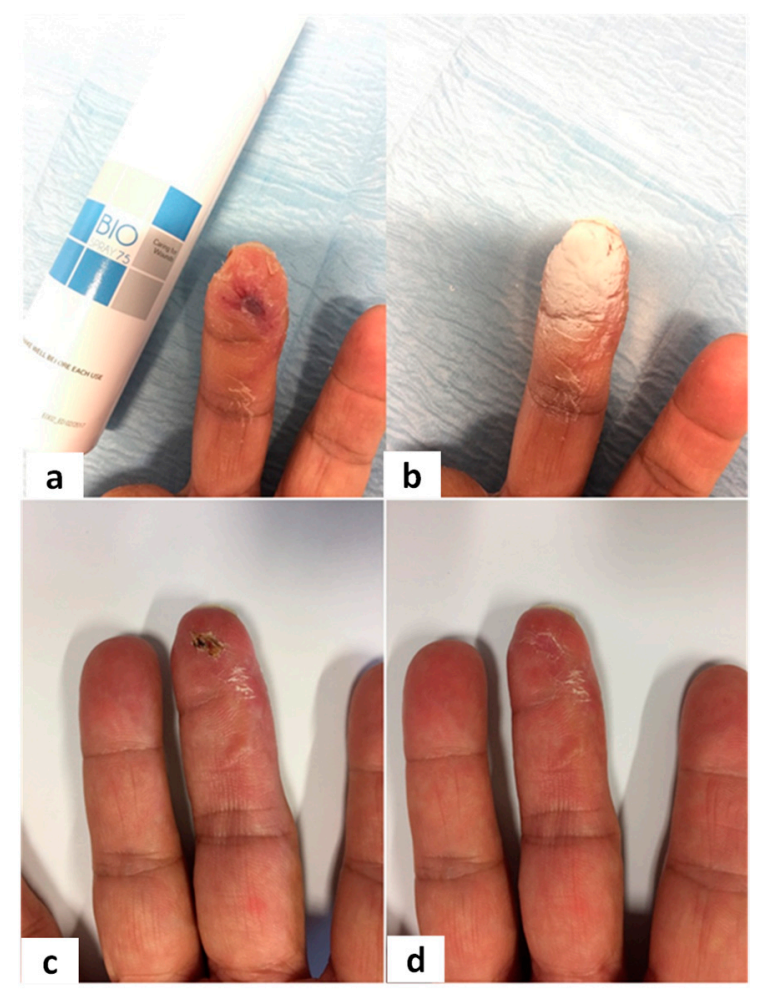

Figure 4. Case Group B. MS, 55 y/o. affected by diabetes and hypertension reported a chronic post-traumatic loss of substance in the volar aspect of the distal phalanx in the middle finger of the left hand. The aspect of the wound 12 weeks after injury (a) at V1. The application of collagen spray (b) in V1. Wound aspect at V4 (c) and final aspect with complete re-epithelialization in V5 (d).
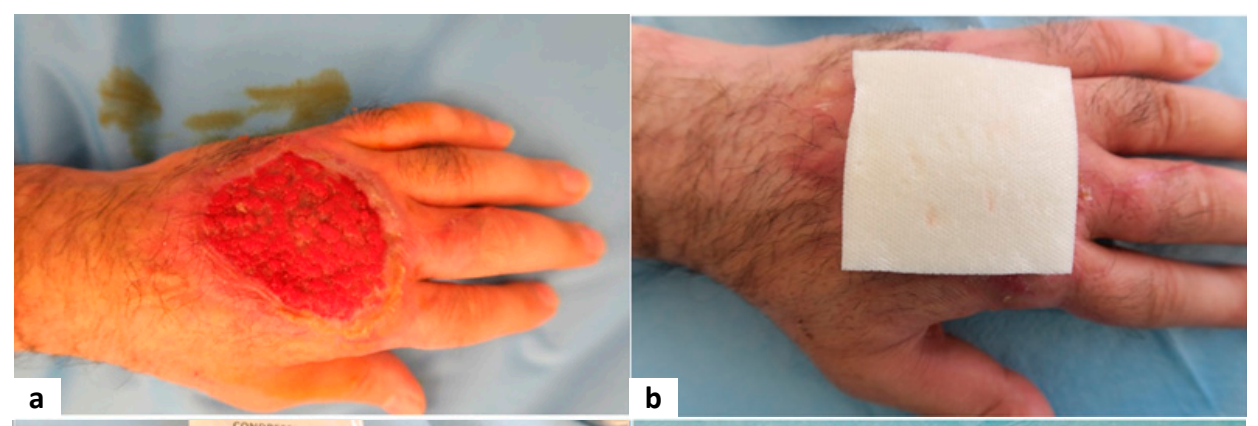

1
6
6
c

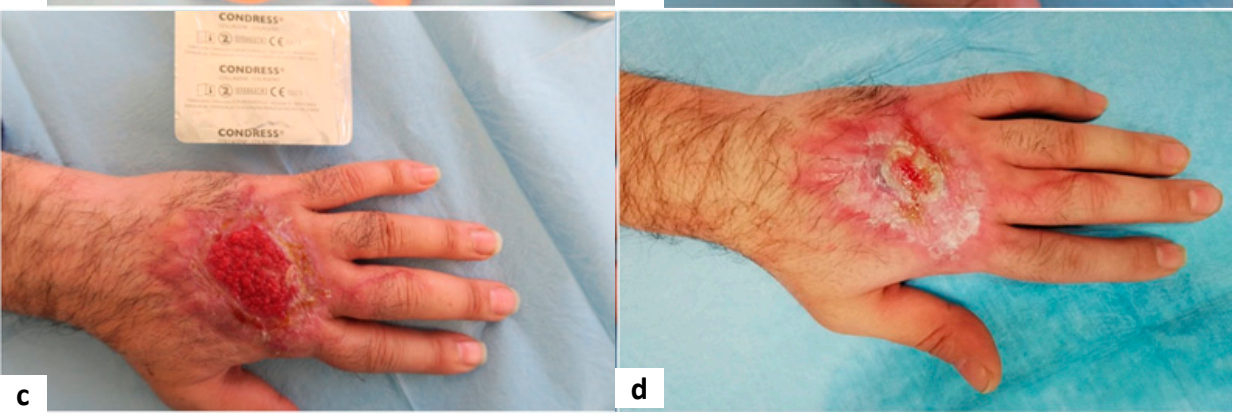

Figure 5. Case Group C. MT male 59 y/o affected by dyslipidemia, diabetes and hypertension reported a post traumatic chronic wound in the dorsal aspect of the left hand. The aspect of the wound 12 weeks after trauma (a). Application in V1 of the collagen plate (b). Wound appearance at V3 (c) and final aspect with almost complete re-epithelialization in V4 (d). 


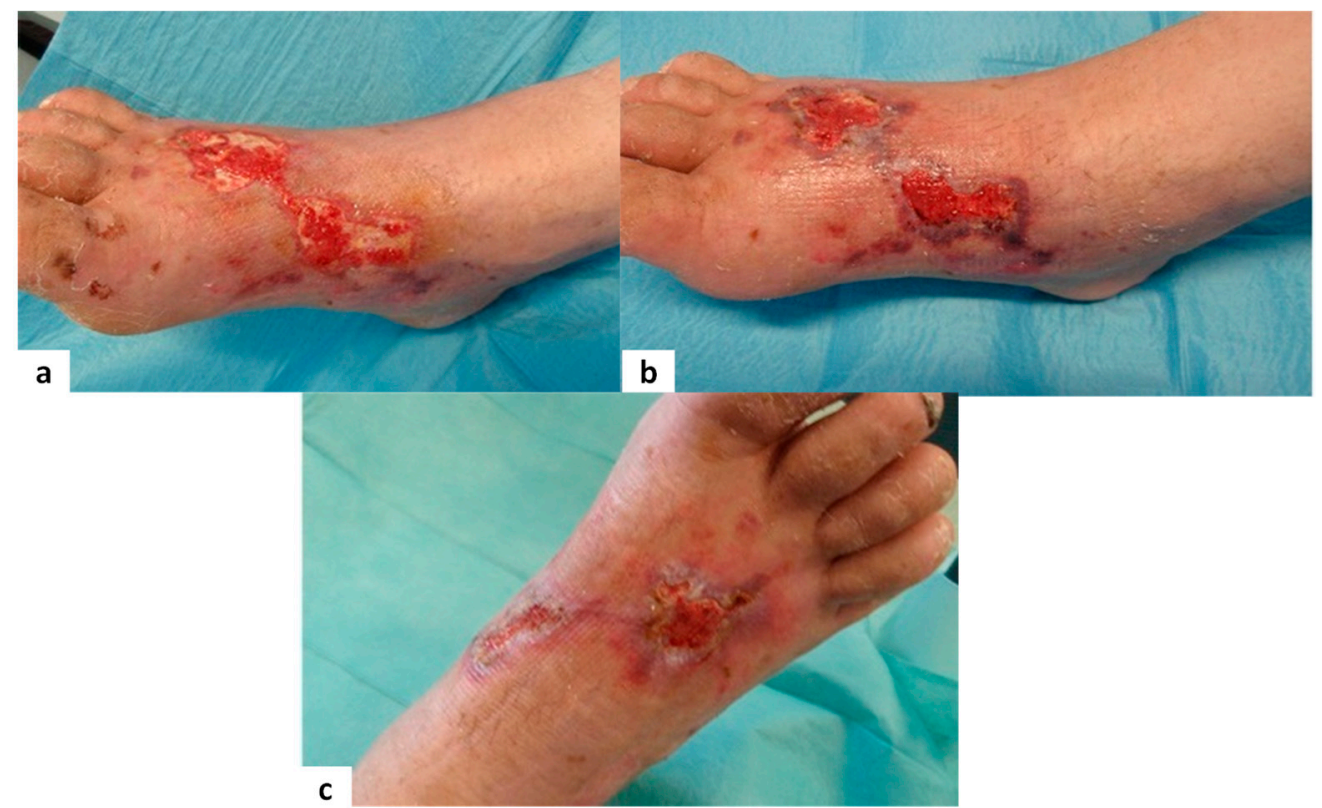

Figure 6. Case Group D. SA, 45 y/o. affected by diabetes and dyslipidemia reported a chronic loss of substance in the dorsal aspect of the right midfoot after crash injury. The aspect of the wound (a) at V1. Wound appearance in V4 (b). Final wound aspect two weeks after V5 (c).

\section{Discussion and Conclusions}

Wound healing is a complex procedure which rapidly generates expression of the various growth factors which promote cellular migration and proliferation and produce new extracellular matrix and collagen depositions $[13,14]$. The increase in metalloproteinase is a common denominator in all chronic wound injuries, and leads to an increase in proteolytic activity and inactivation of the growth factors involved in the healing process. The use of collagen has been shown to specifically inhibit the activity of the proteases without influencing the growth factor function [15]. Furthermore, collagen may be a favourable alternative to the imbued gauze currently considered the standard treatment. This prospective study primarily aimed to assess the efficacy of collagen-based medication as a topical treatment in 128 patients with chronic superficial skin wounds. The secondary objective was to evaluate the safety of collagen medication. All patients were prospectively included for assessment and underwent change of dressing until complete wound recovery for a maximum duration of 28 days.

No statistical differences in recovery were observed between male and female patients in Groups A, B, C and D. Collagen-based therapy was administered to $75 \%$ of patients (divided into three groups), while conventional medication was administered to $25 \%$ of patients. The average wound dimension was $6.49 \pm 3.40 \mathrm{~cm}$ in the collagen-based medication and $8.63 \pm 2.89 \mathrm{~cm}$ in the conventional treatment group. No statistical differences in mean age, wound duration, and wound size were observed between the groups, which most likely was due to the randomized study method. Herein, we report favourable therapeutic effects of collagen-based treatment compared to imbued gauze for wound healing acceleration. In accordance with the literature, we showed that wound healing over time was significantly lower in patients receiving collagen-based treatment compared to conventional treatment [16]. Our results show the efficacy of collagen-based medication to enhance complete wound recovery. However, a major limitation of the randomization of this study is the presence in group B of localized wounds, mainly to the fingers and hands. As a result, our sample may not be fully representative of all chronic wounds. While it is difficult to predict how randomization inclusion would have impacted the findings, evidence from other studies suggests that these findings are in line with the current literature. Donaghue compared the efficacy of alginate-collagen treatment with saline-soaked gauze in 75 patients affected by diabetic foot ulcers [17]. The average re- 
duction percentage of the wound was $80.6 \%$ in patients treated with alginate-collagen and $61.1 \%$ in patients receiving gauze medication. Complete recovery was achieved in $48 \%$ of the alginate-collagen group and $36 \%$ of the gauze medication group. Di Mauro reported that Lyophilized Type I Collagen (LC) was able to considerably improve wound healing in diabetic foot ulcer disorders. The average healing time of the wound in the group treated with LC was $32.4+/-8.6$ days, compared with $49.0+/-11.0$ days in the hyaluronic acid-soaked gauze group. On this basis, our results represent the available assessed evidence on whether collagen is beneficial to wound healing and side effects, and produce evidence for reference in clinical practice of the treatment of wound care.

Furthermore, collagen-based medication is readily absorbed and does not require frequent reapplications, in contrast to conventional medications. However, the ulcer needs to be debrided and cleaned prior to medication application. This study reveals the improved efficacy of the collagen sponge in promoting and stabilizing tissue granulation. The implantable sponge product is dissolved by enzymatic digestion through leukocyte proteases and maintains close contact with the wound bed. Unlike the spray formula, the collagen sponge is strictly involved in the granulation process and plastic-compressed scaffolding to promote fibroblast migration and subsequent endogenous cell invasion. Physiological and natural tissue repair is successfully achieved, and the spongy material supports exudate absorption and inhibits potential extension of the wound, thus preventing bacterial growth which would delay the healing process. Furthermore, the pores are insufficiently large to favour dehydration and insufficiently small to hinder granulation and gas exchange. Heterologous collagen is able to act as a nutritional substrate in the metabolic activity of granulation tissue besides providing mechanical support and cell motility of fibroblasts. The product activation occurs locally without being absorbed by the blood stream, and is not transported into the anatomical systems [18]; on the contrary, it enters the local metabolism of fibroblastic cells and stimulates the production of endogenous collagen essential to wound healing.

Collagen may be considered as a valuable therapeutic tool by virtue of its potential ubiquity, low immunogenicity and flexibility of application in strong and biocompatible scaffolds, and represents a potential advance in wound care. In addition, collagen-based products are able to interface with both natural and synthetic macromolecules. Collagen plays an important role in wound care on account of its ability to activate a controlled release of bioactive molecules. Heterogeneous composites of collagen may be adopted in combination with collagen-mimetic peptides as an influential and dynamic technique to targeted delivery of therapeutic molecules in the wound site, which can in turn accelerate the wound healing process and the regeneration of tissues.

Author Contributions: Conceptualization, F.D.F.; methodology, F.D.F.; validation, M.R.; formal analysis, F.D.F. and M.D.F.; investigation, F.D.F.; resources, M.R.; data curation, M.D.F.; writingoriginal draft preparation, F.D.F.; writing - review and editing, F.D.F. and M.R.; supervision, M.R.; project administration, M.R.; funding acquisition, M.R. All authors have read and agreed to the published version of the manuscript.

Funding: This research was funded by Euroresearch Spa, Milan, Italy, who provided us with the dressings.

Institutional Review Board Statement: The study was conducted according to the guidelines of the Declaration of Helsinki and approved by the Institutional Review Board (or Ethics Committee) of AOU OSPEDALI RIUNITI DI ANCONA (protocol code 2020/56 and date of approval 7 May 2020).

Informed Consent Statement: Informed consent was obtained from all subjects involved in the study. Written informed consent has been obtained from the patients to publish this paper.

Data Availability Statement: The clinical data used to support the findings of this study are included within the article.

Acknowledgments: The authors are grateful to Giuseppina Caraglia, mother-tongue expert, University of Naples "Vanvitelli", for performing English revision. 
Conflicts of Interest: The authors declare no conflict of interest.

\section{References}

1. Sen, C.K.; Gordillo, G.M.; Roy, S.; Kirsner, R.; Lambert, L.; Hunt, T.K.; Gottrup, F.; Gurtner, G.C.; Longaker, M.T. Human skin wounds: A major and snow balling threat to public health and the economy. Wound Repair Regen. 2009, 17, 763-771. [CrossRef]

2. Frykberg, R.G.; Banks, J. Challenges in the treatment of chronic wounds. Adv. Wound Care 2015, 4, 560-582. [CrossRef]

3. Lindley, L.E.; Stojadinovic, O.; Pastar, I.; Tomic-Canic, M. Biology and biomarkers for wound healing. Plast. Reconstr. Surg. 2016, 138, 18S-28S. [CrossRef]

4. Junker, J.P.E.; Kamel, R.A.; Caterson, E.J.; Eriksson, E. Clinical impact upon wound healing and inflammation in moist, wet, and dry environments. Adv. Wound Care 2013, 2, 348-356. [CrossRef]

5. Zelen, C.M.; Serena, T.E.; Denoziere, G.; Fetterolf, D.E. A prospective randomised comparative parallel study of amniotic membrane wound graft in the management of diabetic foot ulcers. Int. Wound J. 2013, 10, 502-507. [CrossRef] [PubMed]

6. Sabo, M.; Le, L.; Yaakov, R.A.; Carter, M.; Serena, T.E. A post-marketing surveillance study of chronic wounds treated with a native collagen calcium alginate dressing. Ostomy Wound Manag. 2018, 64, 38-43. [CrossRef]

7. Lu, P.; Takai, K.; Weaver, V.M.; Werb, Z. Extracellular matrix degradation and remodeling in development and disease. Cold Spring Harb. Perspect. Biol. 2011, 3, a005058. [CrossRef]

8. Tracy, L.E.; Minasian, R.A.; Caterson, E.J. Extracellular matrix and dermal fibroblast function in the healing wound. Adv. Wound Care (New Rochelle) 2018, 5, 119-136. [CrossRef]

9. Udhayakumar, S.; Shankar, K.G.; Sowndarya, S.; Rose, C. Novel fibrous collagen-based cream accelerates fibroblast growth for wound healing applications: In vitro and in vivo evaluation. Biomater. Sci. 2017, 5, 1868-1883. [CrossRef]

10. Gould, L.J. Topical collagen-based biomaterials for chronic wounds: Rationale and clinical application. Adv. Wound Care (New Rochelle) 2016, 5, 19-31. [CrossRef]

11. Gallo, N.; Natali, M.L.; Sannino, A.; Salvatore, L. An overview of the use of equine collagen as emerging material for biomedical applications. J. Funct. Biomater. 2020, 11, 79. [CrossRef]

12. Wiegand, C.; Buhren, B.A.; Bünemann, E.; Schrumpf, H.; Homey, B.; Frykberg, R.G.; Lurie, F.; Gerber, P.A. A novel native collagen dressing with advantageous properties to promote physiological wound healing. J. Wound Care 2016, 25, 713-720. [CrossRef]

13. Singer, A.J.; Clark, R.A. Cutaneous wound healing. N. Engl. J. Med. 1999, 341, 738-746. [CrossRef]

14. Witte, M.B.; Barbul, A. General principles of wound healing. Surg. Clin. N. Am. 1997, 77, 509-528. [CrossRef]

15. Mian, M.; Beghe, F.; Mian, E. Collagen as a pharmacological approach in wound healing. Int. J. Tissue React. 1992, 14, 1-9.

16. Rao, H.; Pai, A.; Hussein, I.; Arun, A.; Shankar Ram, H.; Pai, A.; Pai, S.R.; Pain, S.G. A comparative study between collagen dressings and conventional dressings in wound healing. Int. J. Coll. Res. Int. Med. Public Health 2012, 5, $611-623$.

17. Donaghue, V.M.; Chrzan, J.S.; Rosenblum, B.I.; Giurini, J.M.; Habershaw, G.M.; Veves, A. Evaluation of a collagen-alginate topical wound dressing in the management of diabetic foot ulcers. Adv. Wound Care 1998, 11, 114-119.

18. Chattopadhyay, S.; Raines, R.T. Collagen-based biomaterials for wound healing. Biopolymers 2014, 101, 821-833. [CrossRef] 